\title{
Audit on body mass index in pregnancy
}

This article was published in the following Dove Press journal:

Clinical Audit

12 November 2010

Number of times this article has been viewed

\section{Ratna Aumeer \\ Foundation Year 2 Doctor, Macclesfield District General Hospital, East Cheshire NHS Trust, Mersey Deanery, UK}

Correspondence: Ratna Aumeer Medical Residences Macclesfield District General Hospital, East Cheshire NHS Trust Macclesfield SK 10 3BL United Kingdom $\mathrm{Tel}+447792894$ I 3 I

Email ratna.aumeer@gmail.com
Introduction and background: Obesity during pregnancy is a risk factor for many adverse outcomes such as stillbirth, macrosomia, and gestational diabetes, among others.

Objective: To assess the quality of care and management of obese pregnant women at Dudley Hospital, United Kingdom.

Design: A retrospective audit study.

Setting: Dudley Hospital, Birmingham, United Kingdom.

Standards and criteria: All pregnant women should have their body mass index (BMI) measured and recorded at their first prenatal visit. All pregnant women with BMI $>30$ should have postprandial blood tests at 20 weeks and 26 weeks to screen for diabetes. All pregnant women should receive advice about sensible diet and exercise, which should be documented in their medical notes.

Methods: Medical records for all patients with delivery dates between December 2008 and January 2009 were audited post delivery.

Participants: Pregnant women $(\mathrm{N}=91)$.

Results: BMI is recorded for $98 \%$ of patients. Over a quarter of women with BMI $>30$ did not have postprandial blood tests at 20 and 26 weeks.

Conclusion: BMI is calculated and recorded for most patients, but uptake of postprandial blood tests is suboptimal in patients with BMI $>30$.

Recommendations: To add a section on obesity in the maternity notes.

Keywords: pregnancy, obesity, body mass index (BMI), audit

\section{Introduction and background}

Obesity during pregnancy is a risk factor for adverse pregnancy outcomes. Risks to the baby include stillbirth, macrosomia, and congenital malformations. ${ }^{1}$ Perinatal mortality and risks to the mother include miscarriage, cesarean sections, pre-eclampsia, gestational diabetes, and thromboembolism., ${ }^{2,3}$ Identifying the women with higher BMI, advising them, and regularly consulting them in clinic will hopefully decrease any adverse pregnancy outcomes. ${ }^{4}$

The CEMACH Maternal Death Enquiry on Saving Mothers' Lives found that 27\% of women who died during pregnancy had BMI of $\geq 30$, and, overall, 52\% had BMIs of $\geq 25 .^{5}$ Obesity in pregnancy has been selected to be CEMACH's main project for 2008-2011 and it will seek to evaluate service provision to obese pregnant women throughout the United Kingdom.

Currently, there are no national guidelines in the United Kingdom for care of obese pregnant women. However some hospitals have local protocols in place. The Dudley 
Hospital's NHS Trust Guideline 'Recording Body Mass Index in Pregnancy and Pathway for Referral' was used as a guideline for this project (Appendix 1).

\section{Objectives}

1. To determine whether the current hospital guidelines on the management of pregnant women were being followed at Dudley Hospital.

2. To identify whether new standards needed to be set for the care of pregnant women.

\section{Criteria and standards}

The Dudley Hospital's NHS Trust Guideline (Appendix 1) was used to establish the criteria for this project.

The criteria chosen were:

- All pregnant women should have their BMI calculated at booking or at first antenatal visit.

- All women with a BMI $>30$ should have postprandial blood tests (oral glucose tolerance test) at 20 weeks and again at 26 weeks to screen for diabetes.

- All pregnant women should receive advice about sensible diet and exercise, which needs to be documented in their medical notes.

The standards of these three criteria were set at $100 \%$. However, a more achievable standard needs to be considered during the re-audit phase, particularly the criterion for all women with high BMI to have postprandial blood tests, as some women may refuse or simply not attend for the investigations.

\section{Methods}

An audit questionnaire (Appendix 2) was designed to include the patient's unit number, age, ethnicity, and BMI. BMI was calculated at the time of first antenatal visit, and for some

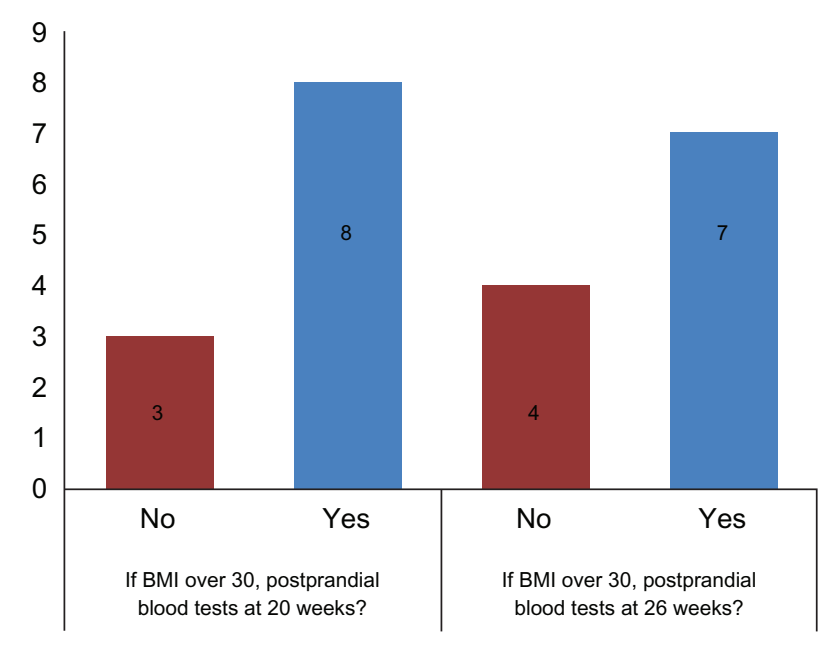

Figure I Postprandial blood test at 20 and 26 weeks. women this can be late during pregnancy. BMI will thus be high in these cases but not necessarily as a result of obesity. The questionnaire also included certain questions about the quality of care of pregnant women, such as if postprandial blood tests were done when required and if advice about diet and exercise was given and documented in the notes. Advice about diet and exercise was given either by midwives or doctors. Patients who had an expected date of delivery during the months of December 2008 and January 2009 were selected for this study. This study was retrospective and each patient was identified and selected after having given birth. The questionnaire was available on the Dudley Hospital Intranet Hub and one questionnaire was filled for each patient. When all the data had been collected, the results were analyzed on an Excel spreadsheet. For the purpose of this study, the section on complications in the proforma was not included in the audit report.

\section{Results}

There were 91 participants, aged between 17 and 42 years old with a median age of 27 years. The two highest ethnic groups were White (75\%) and Asian Pakistani (9\%).

Two out of the 91 participants did not have their BMI calculated at booking or first antenatal visit.

The BMI ranged between 18 and 37. Eleven out of the 91 participants had a BMI $>30$, ie, $12 \%$ of the total population being studied had a BMI $>30$. Out of the 11,3 participants did not have their postprandial blood tests at 20 weeks (27\%) and 4 participants did not have their postprandial tests at 26 weeks (36\%) (Figure 1).

Out of the 91 participants, 20 (22\%) did not have advice about sensible diet and exercise documented in their notes.

For those women with BMI $>30,4$ out of the $11(36 \%)$ did not have the advice documented in their notes.

\section{Discussions and limitations of study}

Of the total population studied, $2 \%$ did not have their BMI recorded. The hospital protocol states that all pregnant

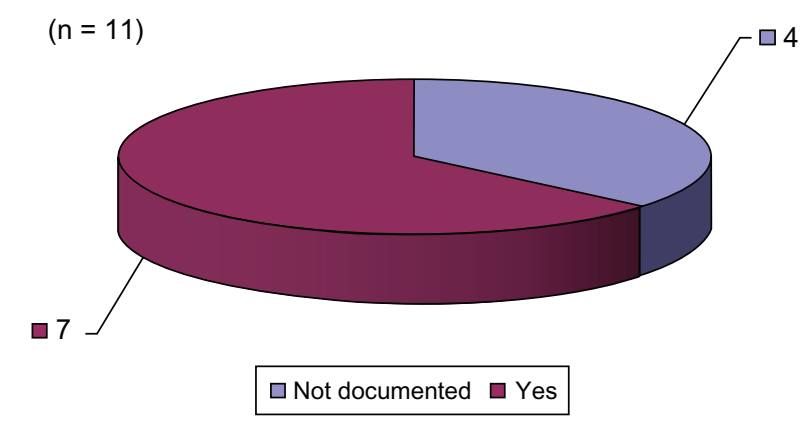

Figure 2 If BMI > 30, advice given about sensible diet and exercise? 
women need to have their BMI calculated and recorded in the patient's notes. This needs to be addressed, as it is a parameter that is simple and quick to calculate.

Of the women with a BMI $>30,27 \%$ did not have postprandial blood tests to screen for diabetes at 20 weeks and $36 \%$ of women with a BMI $>30$ did not have postprandial blood tests at 26 weeks. This is a matter that needs to be addressed, because obese pregnant women have a higher risk of developing diabetes. Two main reasons can be discussed for the low rate of uptake of the screening test. First, health professionals might not be aware that postprandial blood tests need to be done at 20 weeks and again at 26 weeks. This can be altered by having a written note next to the section where BMI is recorded, to remind health professionals of postprandial tests. Second, compliance on the patient's part might be low. Ways to increase the patient's compliance to take the test need to be looked into, such as better health education, leaflets, and reminding the women of the appointment dates.

Of the total population, $22 \%$ did not have advice about sensible diet and exercise documented in their notes and $36 \%$ of women with BMI $>30$ did not have the advice documented in their notes (Figure 2). This needs to be addressed, as the antenatal notes already have a section about lifestyle, diet, and exercise to remind health professionals to discuss these with the patient. Simple verbal advice may have been given and not documented in the notes, so this needs to be considered as well.

\section{Conclusion}

Obesity in pregnancy is well known to be associated with adverse outcomes. This study found that BMI is calculated and recorded for most patients (98\%). However, more than a quarter of women with BMI $>30$ did not receive postprandial blood tests at 20 and 26 weeks. Of women with BMI $>30$, $36 \%$ did not have advice on diet and exercise recorded in their notes. A better pathway of care for obese pregnant women needs to be in place.

\section{Recommendations}

1. Midwives, hospital doctors, and general practitioners can be made aware or reminded of the hospital guidelines through teaching sessions.

2. Health professionals can be advised to document that blood tests have been refused by the patients, and the reasons for refusal.

3. A section on obesity can be added to the maternity notes to remind health professionals of postprandial blood tests that need to be done.

4. Reminders in the form of letters can be sent to women closer to the date of their blood tests.

5. Leaflets about diet and exercise can be handed to patients after clinic appointments if verbal advice has not been given due to lack of time.

6. These changes will be implemented and the study will be re-audited in future.

\section{Disclosure}

The author reports no conflicts of interest.

\section{References}

1. Usha Kiran TS, Hemmadi S, Bethel J, Evans J. Outcome of pregnancy in a woman with an increased body mass index. BJOG. 2005;112: 768-772.

2. Bhattacharya S, Campbell DM, Liston WA. Effect of body mass index on pregnancy outcomes in nulliparous women delivering singleton babies. BMC Public Health. 2007;7:168.

3. Kabiru W, Raynor BD. Obstetric outcomes associated with increase in BMI category during pregnancy. Am J Obstet Gynaecol. 2004;191: 928-932.

4. Krishnamoorthy U, Schram CMH, Hill SR. Maternal obesity in pregnancy: is it time for meaningful research to inform preventive and management strategies? BJOG. 2006;113:1134-1140.

5. CEMACH Saving Mothers' Lives: Reviewing maternal deaths to make motherhood safer 2003-2005. The Seventh Report of the Confidential Enquiries into Maternal Deaths in the United Kingdom. Dec 2007. http://www.cmace.org.uk 


\section{Appendix I}

The Dudley Group of Hospitals NHS Trust Maternity and Children's Services guideline for recording body mass index (BMI) in pregnancy and pathway for referral

- All women should have their BMI calculated at booking or at the first antenatal clinic visit in the community or hospital as part of the full risk assessment.

- Women with a BMI over 35 or under 18 should be referred for Consultant care.

- All women with a BMI over 30 should have postprandial blood tests at 20 weeks and again at 26 weeks to test for diabetes.

- All women should be offered advice about sensible diet and exercise.

- When appropriate and with the woman's consent, referral to the woman's GP or practice nurse should be made, requesting advice for weight management.

- Midwives should be aware of early warning signs of complications, eg, thromboembolism and diabetes, and take appropriate action when necessary.

- All midwives, wards, and departments have been provided with BMI calculators or BMI charts. All women should have their BMI measurement calculated at booking or at the earliest opportunity in pregnancy, and this must be recorded in their notes.

Clinical Audit

\section{Publish your work in this journal}

Clinical Audit is an international, peer-reviewed, open access journal focusing on the processes and outcomes of clinical audit in any area of healthcare. All aspects of patient care are addressed within the journal and practitioners from all disciplines are invited to submit their work. Areas covered include: Publication of audits; How an audit has changed practice;

\section{Appendix 2}

\begin{tabular}{|c|c|c|}
\hline Dudley Group of Hospital & 5 Foundatio & \\
\hline An audit of $B M I$ in pregna & & \\
\hline Unit number & & \\
\hline Age (years) & & \\
\hline Ethnic background & White & Black \\
\hline & Indian & Pakistani \\
\hline & Bangladeshi & Chinese \\
\hline & Other Asian & Other \\
\hline BMI & & \\
\hline BMI calculated at booking & Yes & Not \\
\hline or first antenatal visit & & documented \\
\hline If BMI over 30 , postprandial & Yes & \\
\hline blood tests at 20 weeks & & \\
\hline If BMI over 30 , postprandial & Yes & \\
\hline blood tests at 26 weeks & & \\
\hline $\begin{array}{l}\text { Advice given about sensible } \\
\text { diet and exercise }\end{array}$ & Yes & $\begin{array}{l}\text { Not } \\
\text { documented }\end{array}$ \\
\hline Complications during pregnan & & \\
\hline Miscarriage & Hypertension & \\
\hline Thromboembolism & $\begin{array}{l}\text { Diabetes or in } \\
\text { tolerance }\end{array}$ & glucose \\
\hline Pre-eclampsia & Congenital ab & \\
\hline Anemia & Other & \\
\hline If other, please give details & & \\
\hline Delivery complications? & & \\
\hline Cesarean section & $\begin{array}{l}\text { Instrumental } \\
\text { delivery }\end{array}$ & Other \\
\hline If other, please give details & & \\
\hline Complications to baby? & & \\
\hline Stillbirth & Prematurity & \\
\hline Macrosomia & Congenital $\mathrm{m}$ & ions \\
\hline Hyperglycemia & Other & \\
\hline If other, please give details & & \\
\hline
\end{tabular}

Practical tips on how to do audits and to avoid pitfalls; How audits have changed patient care; Calls and justifications for new audits. The manuscript management system is completely online and includes a very quick and fair peer-review system, which is all easy to use. Visit http://www.dovepress. com/testimonials.php to read real quotes from published authors. 